\title{
Sensors, Medical Images and Signal Processing: Ubiquitous Personalized Health Monitoring
}

\author{
H. Müller ${ }^{1,2}$, A. Foncubierta-Rodriguez ${ }^{2}$, A. Depeursinge ${ }^{1,2}$, Section Editors for the IMIA Year- \\ book Section on Sensor, Signal, and Imaging Informatics \\ 'Medical Informatics, University Hospitals and University of Geneva, Switzerland \\ ${ }^{2}$ University of Applied Sciences Western Switzerland (HES SO), Sierre, Switzerland
}

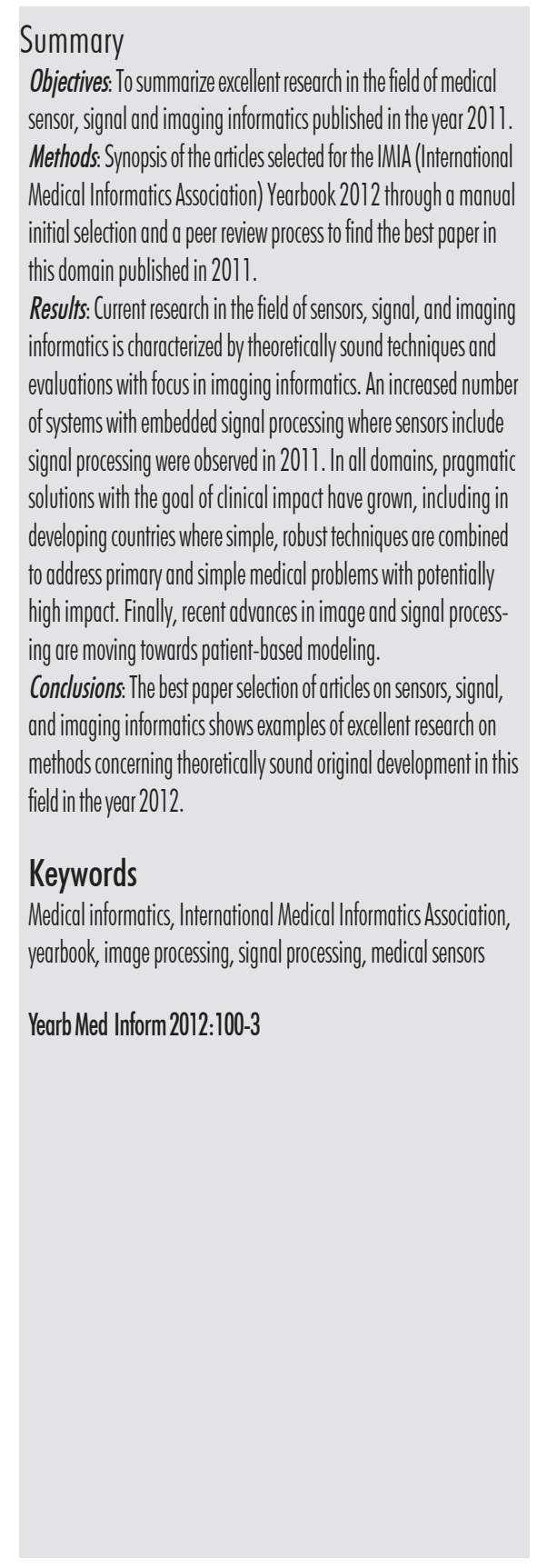

\section{Introduction}

The content of MedLine/Pubmed containing articles published in the year 2011 was searched while hunting for excellent research in the fields of sensors, signals and imaging. Three major trends were observed clearly from the search results:

- sensor-embedded signal processing is becoming increasingly important, aiming at developing autonomous health monitoring,

- pragmatic, simple solutions with high clinical impact are developed, including in developing countries with good engineering departments,

- signal and image processing is increasingly focusing on patient-specific organ modeling.

The research trends are discussed in the following sections and the associated five best papers (after a detailed review process) are listed in Table 1 and summarized in the appendix.

\section{Ubiquitous Embedded Signal Processing for Autonomous Health Monitoring}

The need for portable autonomous health monitoring systems has progressively opened a new domain that results from the fusion of sensors and signals. Much of the signal pre-processing is being done directly by the sensors and the resulting signals rather need interpretation and context than pure signal processing. These outputs can either be cardiac abnormality detection in rural populations [1], multisensor signal aggregation for the understanding of the coordination between locomotion and respiration of Parkinson's disease patients [2], or knowledge discovery to reveal physiological correlates of mental stress [3]. All systems aim at pre-processing and aggregating low-level sensor signals to attract the physician's or researcher's attention on diagnostically relevant events or patterns. Low power consumption, system compactness, data storage, real-time signal processing and inter-sensor communication are all important challenges for such mobile health monitoring devices.

\section{Focusing on the Clinical Impact}

In all domains, a focus on the clinical impact of the developed systems was observed. Mandal et al. [1] put efforts into combining pragmatic solutions to palliate the lack of cardiologists in remote rural areas in India, which results in a portable cardiac sound analysis system to detect heart anomalies and subsequently determines the importance of getting further assistance. Other researchers have systematically analyzed the usability requirements of image-based computer-aided diagnosis systems in clinical routine $[4,5]$ with the aim of reducing the gap between 
systems developed in laboratory and those used in clinical practice.

\section{Patient-specific Organ Modeling}

Recent advances in signal and image modeling fostered the development of personalized organ modeling. The purpose of the latter is to build a reference model for quantifying deviations from patient-specific patterns that are reflecting a subsequent disease, mental state, or treatment response. In [6], Sermesant et al. developed a comprehensive customized electromechanical model of the heart for the prediction of pacing acute effects in cardiac resynchronization therapy (CRT). This model allows for comprehensive in silico studies of the electrical, kinematical and mechanical properties of the heart. Patient-specific assessment of recovery or treatment of knee disorders is based on an anatomical model in magnetic resonance imaging (MRI) in [7]. The model is used to systematically align $2.5 \mathrm{D}$ diagnosis MRI images among 3D follow-up patient scans. In [8], personal liver images (e.g., vessels, tumor and resection planes) are projected onto the liver surface to assist navigation during surgical operations. In [9], patient-specific electroencephalogram (EEG), electrooculogram (EOG) and electrocardiogram (ECG) signal models are built by identifying aggregated signal spectrum components that are related to subject-specific drowsiness during car driving.

\section{Conclusions and Outlook}

The best paper selection for the Yearbook section 'signal, sensor, and imaging informatics' can by no means reflect the broadness of the field. An extremely large number of papers were

Table 1 Best paper selection of articles for the IMIA Yearbook of Medical Informatics 2010 in the section 'Sensor, Signal, and Imaging Informatics'. The articles are listed in alphabetical order of the first author's surname.

Section

Sensor, Signal, and Imaging Informatics

- Choi J, Ahmed B, Gutierrez-Osuna R. Development and Evaluation of an Ambulatory Stress Monitor Based on Wearable Sensors. IEEE Trans Inf Technol Biomed 2012;16(2):279-86.

- Gavaghan KA, Peterhans M, Oliveira-Santos T, Weber S. A portable image overlay projection device for computer-aided open liver surgery. IEEE Trans Biomed Eng 2011;58(6):1855-64.

- Khushaba RN, Kodagoda S, LaI S, Dissanayake G. Driver drowsiness classification using fuzzy wavelet-packet-based feature-extraction algorithm. IEEE Trans Biomed En 2011;58(1):121-31.

- Mandal S, Basak K, Mandana KM, Ray AK, Chatterjee J, Mahadevappa M. Development of cardiac prescreening device for a rural population using ultralow-power embedded system. IEEE Trans Biomed Eng 2011;58(3):745-9.

- Sermesant M, Chabiniok R, Chinchapatnam P, Mansi T, Billet F, Moireau P, Peyrat JM, Wong K, Relan J, Rhode K, Ginks M, Lambiase P, Delingette H, Sorine M, Rinaldi CA, Chapelle D, Razavi R, Ayache N. Patient-specific electromechanical models of the heart for the prediction of pacing acute effects in CRT: A preliminary clinical validation. Med Image Anal 2012;16(1):201-15.

reviewed and even the initial selection of over 100 target articles was hard to make. Reducing this selection to only 20 papers for a detailed review was even harder. The final five articles selected by the peer reviewers represent well the current research trends of the domain of sensors, signals and imaging in medical informatics with dominant research areas in multimodal and multidimensional data $[10,11]$, ubiquitous health monitoring $[1,2,3]$ and personalized organ modeling $[6,7,8,9]$. Up-to-date information about current and future issues of the IMIA Yearbook is available at http://www.schattauer.de/de/ magazine/uebersicht/zeitschriften-a-z/ imia-yearbook/objectives-contentsformats.html.

\section{Acknowledgements}

We greatly acknowledge the support of Martina Hutter and of the reviewers in the selection process of the IMIA Yearbook.

\section{References}

1. Mandal S, Basak K, Mandana KM, Ray AK, Chatterjee J, Mahadevappa M. Development of cardiac prescreening device for rural population using ultralow-power embedded system. IEEE Trans Biomed Eng 2011;58(3):745-9.
2. Ying H, Schlösser M, Schnitzer A, Schäfer T, Schläfke ME, Leonhardt S, et al. Distributed intelligent sensor network for the rehabilitation of Parkinson's patients. IEEE Trans Inf Technol Biomed 2011;15(2):268-76.

3. Choi J,Ahmed B, Gutierrez-Osuna R. Development and Evaluation of an Ambulatory Stress Monitor Based on Wearable Sensors. IEEE Trans Inf Technol Biomed 2012;16(2):279-86.

4. van Ginneken B, Schaefer-Prokop CM, Prokop M. Computer-aided diagnosis: how to move from the laboratory to the clinic, Radiology 2011; 261(3):719-32.

5. Depeursinge A, Fischer B, Müller H, Deserno TM. Prototypes for content-based image retrieval in clinical practice. Open Med Inform J 2011; 5(Suppl 1):58-72.

6. Sermesant M, Chabiniok R, Chinchapatnam P, Mansi T, Billet F, Moireau P, et al. Patient-specific electromechanical models of the heart for the prediction of pacing acute effects in CRT: A preliminary clinical validation. Med Image Anal 2012;16(1):201-15

7. Zhan Y, Dewan M, Harder M, Krishnan A, Zhou XS. Robust automatic knee MR slice positioning through redundant and hierarchical anatomy detection, IEEE Trans Med Imaging 2011; 30(12):2087-100

8. Gavaghan KA, Peterhans M, Oliveira-Santos T, Weber S. A portable image overlay projection device for computer-aided open liver surgery. IEEE Trans Biomed Eng; 58(6):1855-64, 2011.

9. Khushaba RN, Kodagoda S, Lal S, Dissanayake G, Driver drowsiness classification using fuzzy waveletpacket-based feature-extraction algorithm, IEEE Trans Biomed Eng 2011;58(1):121-31.

10. Madabhushi A, Agner S, Basavanhally A, Doyle S, Lee G. Computer-aided prognosis: predicting patient and disease outcome via quantitative fusion of multi-scale, multi-modal data. Comput Med 
Imaging Graph 2011;35(7-8):506-14

11. Padilla P, López M, Górriz JM, Ramírez J, SalasGonzález D, Álvarez I; Alzheimer's Disease Neuroimaging Initiative. NMF-SVM based CAD tool applied to functional brain images for the diagnosis of Alzheimer's disease. IEEE Trans Med Imaging 2012;31(2):207-16.

12. Iyer VK, Ramamoorthy PA, Fan H, Ploysongsang Y. Reduction of heart sounds from lung sounds by adaptive filtering. IEEE Trans Biomed Eng 1986; 33(12):1141-8.

Correspondence to:

Prof. Dr. Henning Müller

HES SO Valais

TechnoArk 3, 3960 Sierre

Switzerland

E-mial: henning.mueller@hevs.ch

Appendix: Content Summaries of Selected Best Papers for the IMIA Yearbook 2012, Section Sensors, Signal and Imaging Informatics*

\section{Choi J, Ahmed B, Gutierrez-Osuna R \\ Development and Evaluation of an Am- bulatory Stress Monitor Based on Wear- able Sensors. \\ IEEE Trans Inf Technol Biomed 2012;16(2):279-86}

Choi et al. combine recent wearable sensor technologies with mobile computing to monitor stress on an ambulatory basis. Heart and respiration rate, skin conductance and electromyography of the trapezius muscle are continuously recorded by a holster unit, which also includes a 3D accelerometer and a global positioning system (GPS) chip. The whole system is designed to be minimally disruptive to avoid any influence of the monitoring device on the activities of the subject. Sensor-specific signal processing and logistic regression

* The complete papers can be accessed in the Yearbook's full electronic version, provided that the article is freely accesible or that your institution has access to the respective journal. are used to discriminate between mental stress and relaxation. Electrodermal activity is first detrended using a second-order derivative operator to be decomposed into an aperiodic trend and a residual. The mean and correlation with time of the aperiodic trend and the standard deviation of the residual are used as electrodermal features. Another feature is derived from heart and respiration rate using the ratio of the normalized power spectral density of the parasympathetic and the sympathetic nervous systems. A validation of the system with 10 subjects that were asked to perform a set of five mental stress activities interlaced by relaxation periods showed an average classification rate of $81 \%$.

\section{Gavaghan KA, Peterhans M, Oliveira-Santos T, Weber $S$ \\ A portable image overlay projection device for computer-aided open liver surgery IEEE Trans Biomed Eng 2011;58(6):1855-64}

In [8], Gavaghan et al. describe an innovative navigation system for open liver surgery. A portable image overlay projection device is developed to display patient-specific liver model images (e.g., vessels, tumor and resection planes) on the liver surface. It allows for a systematic correspondence between structures that were previously identified in computer-aided visualization software and the actual view of the liver in the operating room. The navigation system includes a portable laser projector, a video processor and a microelectromechanical system controller. After a calibration phase based on a projected checkerboard, the projected image automatically adapts to the position and orientation of the portable projector. The projection accuracy is evaluated using a 2D checkerboard and a 3D liver model and was estimated to have a mean error of 1.3 millimeters.

\section{Khushaba RN, Kodagoda S, Lal S, Dissanayake G}

Driver drowsiness classification using fuzzy wavelet-packet-based feature-extraction algorithm

IEEE Trans Biomed En 2011;58(1):121-31

A system for monitoring driver drowsiness is proposed by Khushbaba et al. in [9]. An aggregation of signals from electroencephalogram, electrooculogram and electrocardiogram is carried out to predict the driver drowsiness on a five-step scale. Wavelet packet transforms optimized using fuzzy mutual information are used to extract features from each signal source. The optimal subset of wavelet packet subbands that maximizes fuzzy mutual information is further used by a classification algorithm to predict the drowsiness level of the driver. This has the advantage of not restricting the spectral analysis to well-known $\alpha, \beta, \theta$ or $\bar{\sigma}$ bands but rather to identify spectrum components that are related to subject-specific drowsiness. The validation of the methods is based on 31 subjects that were asked to perform two driving sessions on a simulator. The driving sessions are segmented into various tracks with varying traffic intensity levels. The level of drowsiness was estimated based on state-of-the-art criteria from the driver's face such as facial tone, slow eyelid closure and several mannerisms of the driver.

\section{Mandal S, Basak K, Mandana KM, Ray AK, Chatterjee J, Mahadevappa M \\ Development of cardiac prescreening device for a rural population using ultralow-power embedded system}

\section{IEEE Trans Biomed Eng 2011;58(3):745-9}

A cardiac prescreening device for the rural population is proposed by Mandal et al. [1]. The embedded system analyses heart sounds to provide a preliminary indicative diagnosis of cardiac conditions such as normal, abnormal, ischemic or with valvular abnormalities. The hardware implementation allows for minimal power consumption by adapting the clock of the central 
processing unit (CPU) based on the requirements of the running application. An estimation of the background wideband noise is first removed from the audio signal from the microphone using an adaptive line enhancer [12]. Then, wavelet analysis is used both for denoising and to extract signal peaks for further frequency analysis. A validation of the system using 72 samples of 17 volunteers showed classification performance that is comparable to a cardiologist for all classes, except for undetermined abnormal cases. Such a portable monitoring device is therefore promising to assess primary heart disorders in rural areas where cardiologists are rare.

\section{Sermesant M, Chabiniok R, Chinchapatnam P, Mansi T, Billet F, Moireau P, Peyrat JM, Wong K, Relan J, Rhode K, Ginks M, Lambiase P, Delingette H, Sorine M, Rinaldi CA, Chapelle D, Razavi R, Ayache N \\ Patient-specific electromechanical models of the heart for the prediction of pacing acute effects in CRT: A preliminary clinical validation \\ Med Image Anal 2012;16(1):201-15}

In [6], Sermesant et al. introduce a biophysical model of the human heart to predict the response to cardiac resynchronization therapy (CRT). The model can be personalized in terms of anatomical, electrophysiological, kin- ematic and mechanical properties in order to estimate the acute hemo-dynamic changes associated with CRT. The model uses anatomical MRI to personalize anatomical properties, data from an endocardial catheter to derive electrophysiological properties, a cineMRI for estimating ventricular kinematics, and a pressure catheter to establish mechanical properties. A validation of the model on two clinical cases shows adequacy to invasive hemodynamic measurements with a maximum of $5 \%$ of error on the prediction of the pressure time-derivative. 